\title{
Hydrochloric acid-enhanced radiofrequency ablation for treating a large hepatocellular carcinoma with spontaneous rapture: a case report
}

\author{
Jin-Hua Huang ${ }^{1 *}$, John N. Morelli², Fei Ai ${ }^{1}$, Ru-Hai Zou' ${ }^{1}$, Yang-Kui Gu', Fei Gao' , Tian-Qi Zhang ${ }^{1}$, Wang Yao ${ }^{3}$,
} Xiong-Ying Jiang ${ }^{4}$ and Yan-Yang Zhang ${ }^{5}$

\begin{abstract}
Background: A ruptured hepatocellular carcinoma (HCC) is often fatal. In addition to surgery and transarterial embolization, radiofrequency ablation (RFA) might be another option for treating a ruptured HCC. Unfortunately, conventional RFA has a limited ablation zone; as such, it is rarely used to treat ruptured tumors.

Case presentation: This case was a 60-year-old man who had a large, ruptured HCC in which hydrochloric acid ( $\mathrm{HCl}$ )-enhanced RFA successfully controlled the bleeding and made the tumor completely necrotic.

Conclusion: Considering the effectiveness of HCl-enhanced RFA in achieving hemostasis and tumor ablation, it might be a new option for treating large, ruptured HCCs.
\end{abstract}

Keywords: Hydrochloric acid, Radiofrequency ablation, Spontaneous rupture, Hepatocellular carcinoma

\section{Background}

Liver injuries are common and often result in abdominal pain, bleeding, hypotension, and other conditions requiring immediate attention. Hepatic hemorrhage most commonly occurs because of blunt trauma or the spontaneous rupture of hepatic neoplasms, including hepatocellular carcinoma (HCC), adenoma, focal nodular hyperplasia, and hemangioma.

In Asia and Africa, the most common neoplastic cause of hepatic hemorrhage is HCC, owing to the high prevalence of the disease [1]. The occurrence rate of spontaneously ruptured HCC ranges from $3 \%$ to $14.5 \%$; a ruptured $\mathrm{HCC}$ with intraperitoneal hemorrhage is a life-threatening complication with a 30-day hospital mortality between $31 \%$ and $67 \%[2,3]$. Several factors are

\footnotetext{
${ }^{*}$ Correspondence: huangjh@sysucc.org.cn

1 Department of Minimally Invasive Interventional Therapy, Sun

Yat-sen University Cancer Center, State Key Laboratory of Oncology

in South China, Collaborative Innovation Center for Cancer Medicine, Guangzhou 510060, Guangdong, P. R. China

Full list of author information is available at the end of the article
}

associated with hemorrhagic rupture of $\mathrm{HCC}$, including trauma, large tumor size, vascular invasion, multiple nodules, prior transarterial chemoembolization (TACE), portal venous hypertension, and vascular injury $[3,4]$. Hepatic hemorrhage related to a ruptured $\mathrm{HCC}$ can rapidly cause hemodynamic instability that requires emergency treatment.

Treatment options for ruptured hemorrhagic HCC include packing, plication, emergent hepatic artery ligation, hepatectomy, transarterial embolization (TAE), and radiofrequency ablation (RFA) $[3,5,6]$. In some cases, TAE and surgery are not effective. Moreover, a 3-cm ablative zone produced by a single RFA probe or a $5-\mathrm{cm}$ ablative zone obtained with a cluster of probes is not sufficient to ablate a large tumor. However, hydrochloric acid ( $\mathrm{HCl})$-enhanced RFA has a much larger ablation zone, making it potentially suitable for controlling bleeding from a large, ruptured HCC. 


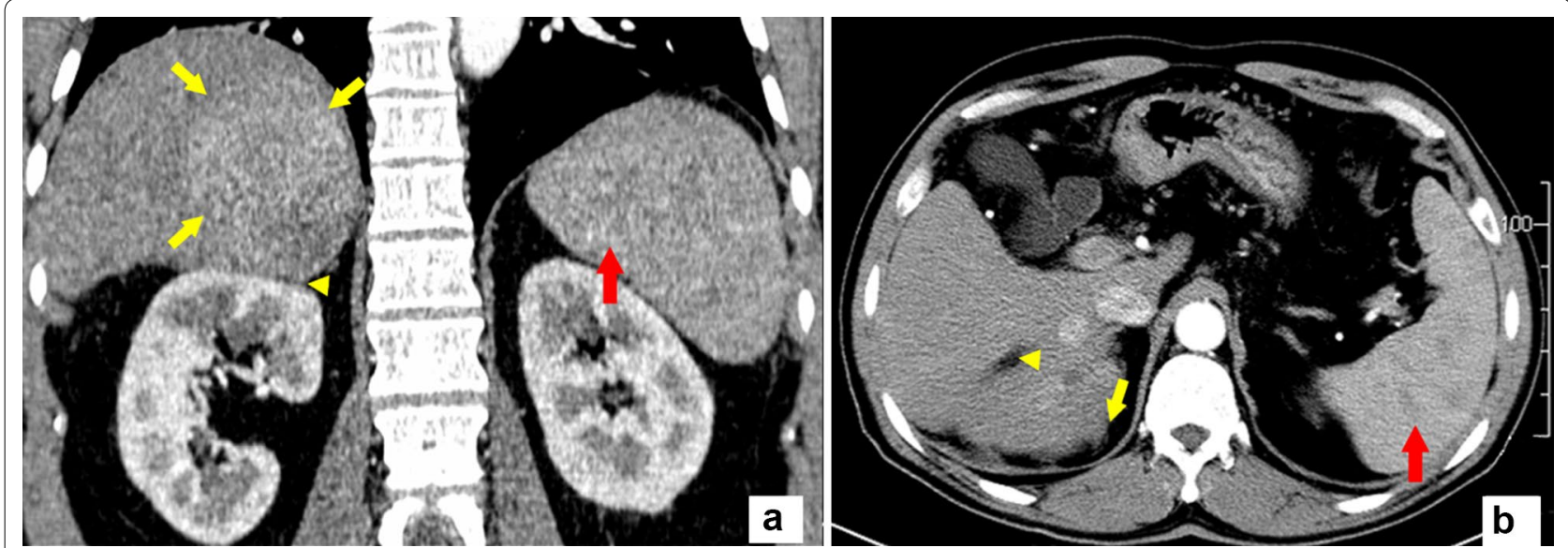

Fig. 1 The initial computed tomography (CT) images of the patient with large hepatocellular carcinoma. The coronal $\mathbf{a}$ and axil $\mathbf{b}$ CT images in the arterial phase show a tumor $(5.8 \mathrm{~cm} \times 7.6 \mathrm{~cm}$ ) occupying liver segments VI-VII (yellow arrows) with multifocal lesions. The inferior portion of the tumor protrudes through the liver contour adjacent to the ipsilateral renal fascia (yellow arrowhead). Splenomegaly can be seen as well (red arrow)

\section{Case report}

On December 13, 2013, a 60-year-old man presented at Sun Yat-sen University Cancer Center with a diagnosis of HCC (Barcelona Clinic Liver Cancer stage B; Fig. 1), severe cirrhosis, and splenomegaly. On January 9, 2014, because of his impaired liver function (evaluated as Child-Pugh B) and low platelet count, he underwent TACE and a partial spleen embolization (PSE). A subsequent contrast-enhanced computed tomography (CT) scan taken on February 27, 2014 revealed a tumor with compact deposition in the upper portion and suboptimal deposition in the lower portion (Fig. 2). Moreover, his platelet count increased from $45.0 \times 10^{9} / \mathrm{L}$ at baseline to $115.0 \times 10^{9} / \mathrm{L}$ after PSE, which is in the normal range, and his carbohydrate antigen concentration decreased from 216.1 to $111.9 \mathrm{U} / \mathrm{mL}$. As the patient had Child-Pugh B liver function and moderate anemia, we therefore thought that he could tolerate a second TACE.

On March 15, 2014, after pre-TACE workup, the patient experienced sudden abdominal pain following a hard defecation. Based on obvious signs of peritoneal irritation and uncoagulated hemorrhagic fluid in the perihepatic region obtained under ultrasound-guided abdominal paracentesis, a diagnosis of a spontaneously ruptured HCC was confirmed. An emergent TAE was performed by the physicians on duty. Digital subtraction angiography (DSA) showed that a large amount of
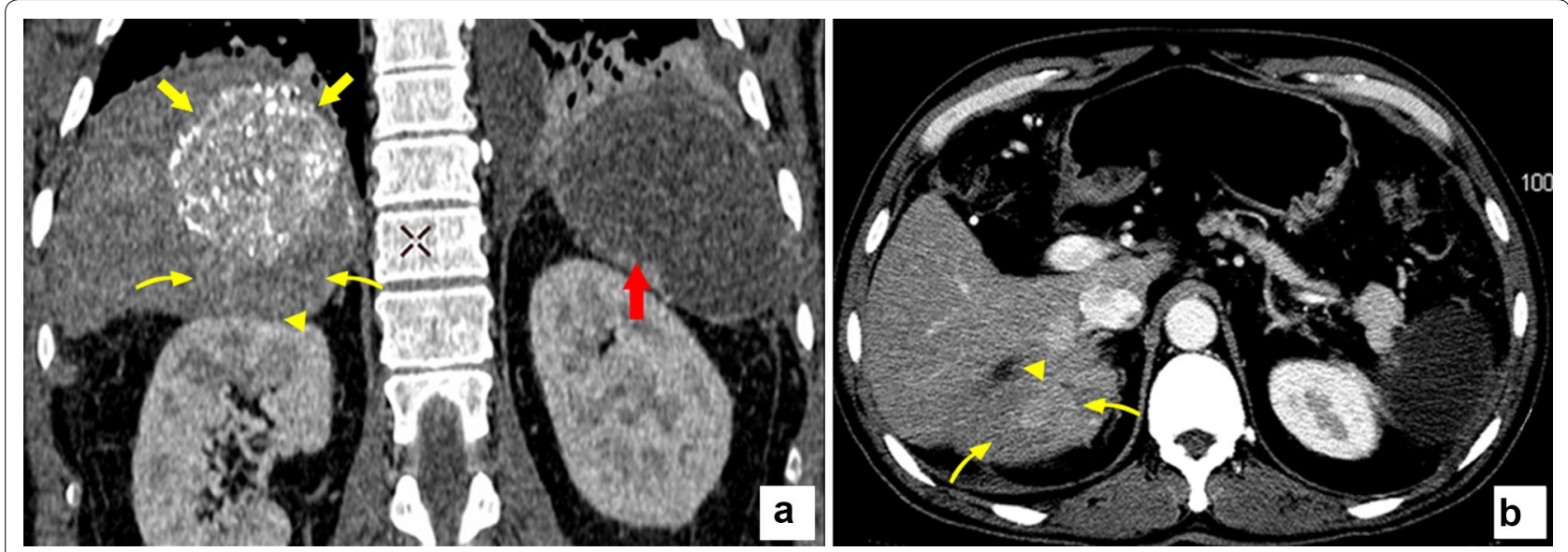

Fig. 2 The follow-up CT images after transarterial chemoembolization (TACE). The coronal $\mathbf{a}$ and axil $\mathbf{b}$ CT images show the tumor with inhomogeneous deposition of lipiodol, the compact upper portion (yellow arrow) and the lower portion (yellow curved arrow) without lipiodol deposition which was still enhanced in the arterial phase. The protruded tumor was located adjacent to the upper polar of the right kidney (yellow arrowhead). The spleen was inactive after partial embolization (red arrow) 


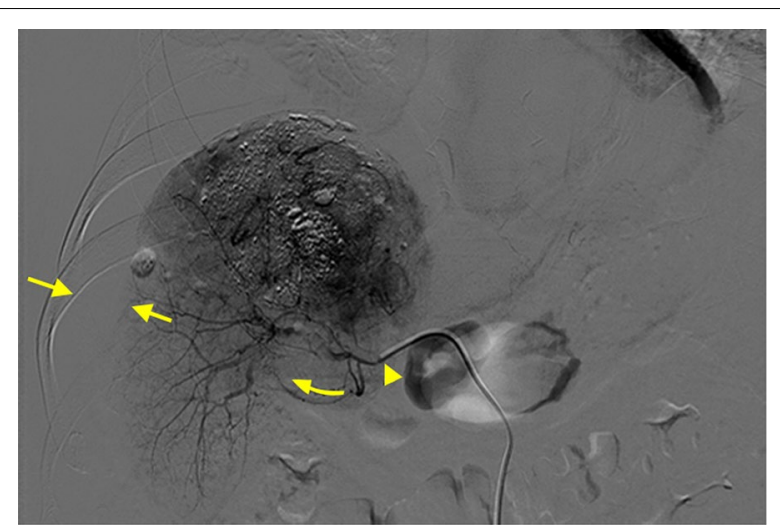

Fig. 3 The digital subtraction angiography image of the emergent transarterial embolization for arresting bleeding. No lipiodol deposition is observed in the upper portion of hepatocellular carcinoma (curved arrow). The feeding arterial vessel is difficult to trace (arrowhead). The crumpled hepatic parenchyma is separated from the abdominal wall by the hemoperitoneum (twin arrows)

intraperitoneal hemorrhage had pushed the liver parenchyma away from the abdominal wall (Fig. 3). Unfortunately, the patient became hemodynamically unstable during the next $12 \mathrm{~h}$, and increasing ascites and active bleeding were confirmed by ultrasound. His liver function deteriorated to Child-Pugh $\mathrm{C}$, making surgery inappropriate. Considering several reports of ruptured HCC being controlled by emergent RFA and our experience with ablation-expanding, $\mathrm{HCl}$-enhanced RFA, we decided to try to control his bleeding with $\mathrm{HCl}$-enhanced RFA [6, 7].

Written informed consent to the procedure and consent to publish were obtained from the patient and his family. The $\mathrm{HCl}$-enhanced RFA procedure was approved by the Clinical Research Ethics Committee of Sun Yatsen Cancer Center.

A large ablative zone can be achieved with $\mathrm{HCl}-$ enhanced RFA by constantly infusing $\mathrm{HCl}$ at a rate of $0.2 \mathrm{~mL} / \mathrm{min}$ and simultaneously delivering radiofrequency (RF) energy with an RF generator (Model 1500X, AngioDynamics, RITA Medical Systems, Queensbury, NY, USA) coupled with a monopolar perfusion electrode (UniBlate, RITA Medical Systems). We used 10\% $\mathrm{HCl}$ (Huayi Medical Auxiliary Materials Manufacturing Co., Ltd, Chengdu, Sichuan, China) as the perfusate, which was diffused in the center of the tumor. $\mathrm{HCl}$ is thought to increase the ionic concentration around the electrode and delay charring of the surrounding tissue [7]. With the patient under local anesthesia, $\mathrm{HCl}$-enhanced RFA was applied for $100 \mathrm{~min}$ with a constant stable output power of $100 \mathrm{~W}$ and a temperature of $103{ }^{\circ} \mathrm{C}$ to the potential bleeding site, which was probably the portion of the protruding liver contour without any lipiodol deposition (Fig. 4).

After CT-guided, HCl-enhanced RFA, the patient's vital signs returned to normal within $24 \mathrm{~h}$. The peritoneal drainage fluid changed from hemorrhagic dark red to light yellow, which confirmed control of the bleeding. Additional conservative treatments were administered during follow-up. Moreover, magnetic resonance imaging performed 1, 6, and 9 months later showed a necrotic tumor with little liquid surrounding the hepatic capsule (Fig. 5). By the end of October 2015, the patient had been alive for 16 months and had an acceptable quality of life, with Child-Pugh B liver function.

\section{Discussion}

Spontaneous tumor rupture is a potential consequence of $\mathrm{HCC}$ and results in a high mortality [3]. The most common mechanisms for hemoperitoneum in HCC rupture are tumor pseudo-capsule disruption and tearing of a parasitic feeding artery. Kanematsu et al. [8] hypothesized that several features visible on CT images portend HCC rupture: a large tumor size, an area of protruded tumor, and a large extent of contact between the tumor and the liver surface. Consistent with this hypothesis, the large HCC in this case eventually ruptured.

Hemostasis is the primary consideration in HCC rupture, and adequate bleeding controlling is vital to patient prognosis. As treatment opinions regarding ruptured HCC have evolved and as new clinical technologies have been adopted, treatment strategies for ruptured HCC have become increasingly diverse, including various conservative, surgical, and minimally invasive measurements. In 1965, Ong et al. [9] advocated packing the ruptured liver as an efficient method to control hemorrhage; then, in 1972, they advocated ligating the hepatic artery. However, with simple packing, they found a high rate of re-bleeding. Moreover, in some cases in the 1970s, direct plication by overlying the capsule was determined to achieve hemostasis [8]. Because the results were disappointing, though, given the friability of $\mathrm{HCC}$, this method is now seldom used. Although emergent hepatectomy remains the most effective treatment for ruptured $\mathrm{HCC}$, it accounts for $7 \%$ of the hospital mortality [3].

With the increasing application of interventional techniques in the last 20 years, TAE emerged as another procedure that could potentially achieve hemostasis in a ruptured HCC, having a success rate between $53 \%$ and $100 \%$ and resulting in a lower 30-day hospital mortality $(0 \%-37 \%)$ than those of surgery [5]. The advantages of TAE over surgery are that it is minimally invasive and directly occludes the feeding vessels, which means that surgery can be avoided in patients at high risk. 


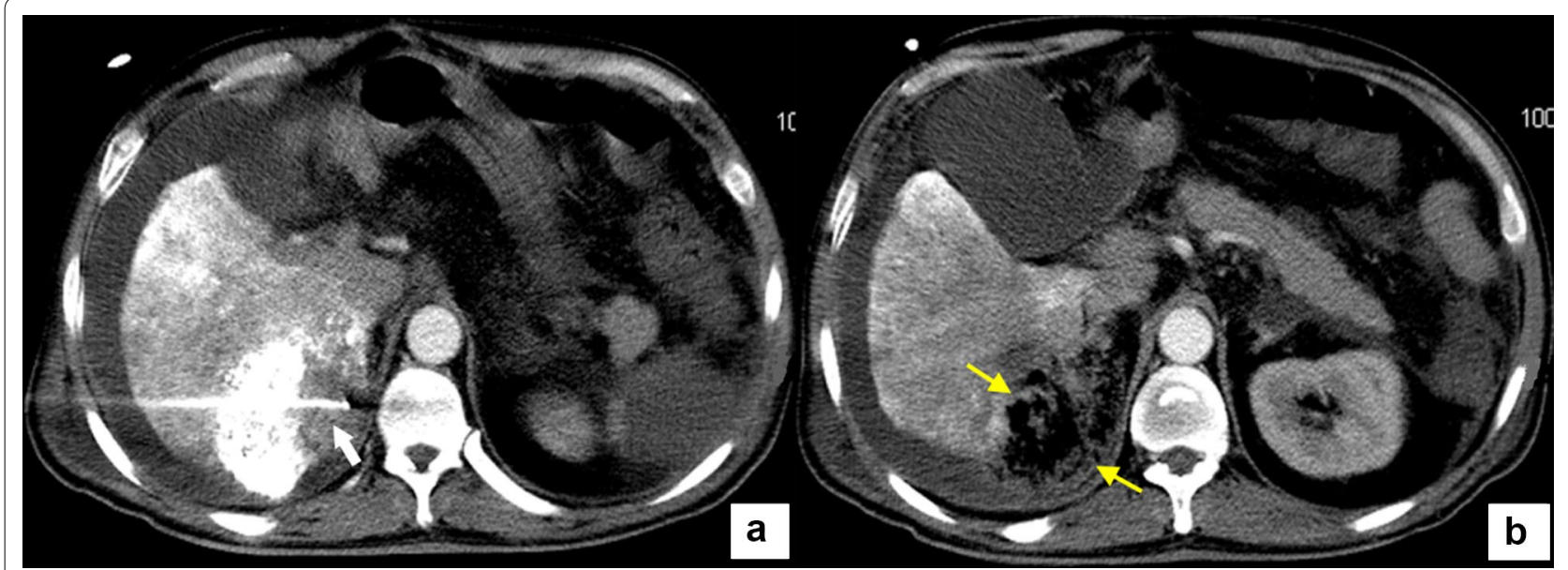

Fig. 4 The CT images of the hydrochloric acid ( $\mathrm{HCl}$ )-enhanced radiofrequency ablation (RFA) procedure. a The RFA electrode (arrow) is inserted into the portion without lipiodol deposition. $\mathbf{b}$ The loose necrotic HCC lesion after HCl-enhanced RFA procedure hangs on the renal region (twin arrows)
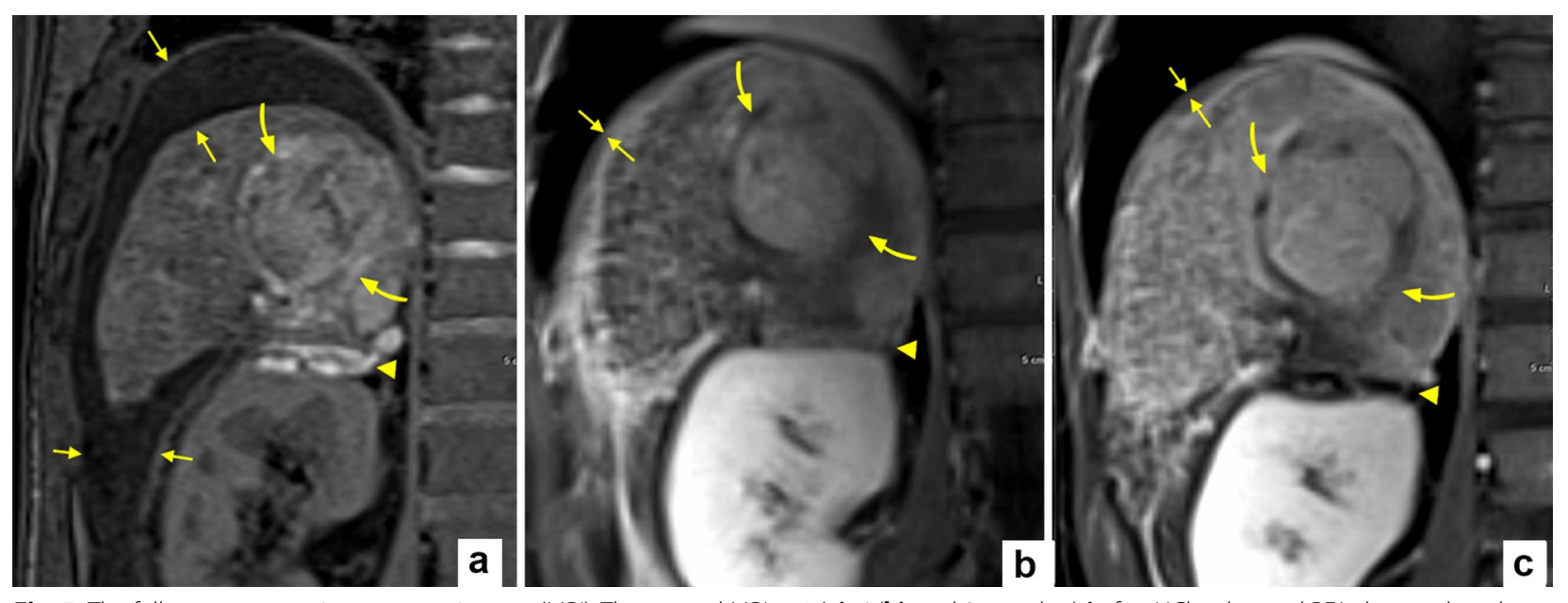

Fig. 5 The follow-up magnetic resonance images (MRI). The coronal MRI at 1 (a), 6 (b), and 9 months (c) after HCl-enhanced RFA show reduced lesion size and a satisfactory tumor capsule beyond the hepatic contour (curved arrow). The gradually distinct margin of the upper renal fascia is also visible (arrowhead). Perihepatic and perirenal ascites are decreased (twin arrows)

Recently, several studies reported successful hemostasis using RFA $[6,10]$. In addition, Chueng et al. [1] found that RFA is safe and effective for achieving hemostasis in ruptured HCC with satisfactory survival outcomes, and therefore they proposed RFA as a first-line option for treating a ruptured HCC. However, needle placement with laparoscopic RFA is less accurate than that with image-guided percutaneous RFA, which has particular advantages for treating lesions within the deep hepatic parenchyma.

In the present case, because our patient had severe cirrhosis, he could not tolerate surgery. Moreover, a failed TAE impaired his hepatic function. As Leung et al. [11] reported, non-super-selective TAE as regional therapy is harmful to patients. They thought that there was typically only one opportunity to achieve hemostasis with TAE, and that more than one attempt would harm the normal hepatic parenchyma.

When the tumors are large or peripherally located, extrahepatic collateral arteries commonly supply HCCs [12]. In our case, both enhanced CT scanning and DSA revealed a filling defect on the lower portion of the tumor. Thus, considering the location of the nonenhanced tumor, collateral vessels supplying this portion were probably branches of the adrenal artery. 
After TAE failed to restore hemostasis, and considering both the patient's overall condition and the results of our previous clinical studies with $\mathrm{HCl}$-enhanced RFA, we determined that CT-guided RFA was a reasonable treatment option. A prior PSE had increased the patient's platelet count to the level on which coagulation was adequate to proceed with RFA [13]. However, currently, the maximum ablative zone obtainable with conventional monopolar RFA is $3 \mathrm{~cm}$ in diameter. Thus, in this case, the 7 -cm diameter tumor would be difficult to ablate in one session. Additionally, our previous experiments showed that infusing diluted $\mathrm{HCl}$ instead of natural saline into the ablation zone during RFA with monopolar perfusion electrodes could enlarge the ablation size (mean \pm standard deviation) from $3.49 \pm 0.07$ to $6.85 \pm 0.32 \mathrm{~cm}$ under the same power and time settings (30 W and $30 \mathrm{~min}$ ) [14]. Given these considerations and the patient's large hepatic mass, we decided to use $\mathrm{HCl}$-enhanced RFA instead of natural saline-infused RFA. In our case, $\mathrm{HCl}$-enhanced RFA damaged neither the nearby duodenum nor the renal fascia. This result may relate to the progressive decrease in temperature from the center to the periphery and to the insulating effects of the fibrous fascia.

In contrast to normal saline, $\mathrm{HCl}$ functions as a chemical ablation agent and is capable of producing an ablative lesion itself [15]. Furthermore, $\mathrm{HCl}$ is a strong electrolyte whose conductivity is about three times that of natural saline, which can increase the conductivity of RF energy. Thus, when $\mathrm{HCl}$ is instilled around the RF electrode, the $R F$ energy expands from a needle shape into a spherical shape. This enlarged spherical volume increases contact area between the RF source and the tissue intended for ablation. In turn, this increased contact area transfers and sustains RF energy much longer (100 min in this case) than normal saline, lengthening the charred area and prolonging the delivery of energy, thus creating a larger ablation zone than any other current RFA method. Therefore, our success in treating this ruptured HCC suggests that a treatment pattern of $\mathrm{HCl}$-enhanced RFA followed by TAE or combined with surgery could be considered in the future.

As in the present case, for patients with a cirrhotic liver, $\mathrm{HCC}$ is often associated with survival of less than 30 days [3]. However, our patient has survived for 16 months. Treatment with $\mathrm{HCl}$-enhanced RFA achieved immediate and total hemostasis and completely ablated the large, bleeding tumor. In addition, as a regional therapy, $\mathrm{HCl}$ enhanced RFA resulted in well-preserved liver function; thus, the patient could recuperate rapidly.

In conclusion, $\mathrm{HCl}$-enhanced RFA appears to be a safe and effective treatment for controlling bleeding after spontaneous rupture of large HCC. However, further research is necessary to confirm our findings.

\section{Authors' contributions}

$J H H, Y K G, F G, W Y$, and $Y Y Z$ were responsible for the patient's treatment. FA, $\mathrm{RHZ}, \mathrm{YKG}$, and FG collected the patient's information. TQZ, WY, and XYJ mainly searched the references for this manuscript.JHH, JNM, TQZ, and FA drafted this manuscript. JHH, JNM, and YKG offered constructive suggestions for this study. All authors read and approved the final manuscript.

\section{Author details}

${ }^{1}$ Department of Minimally Invasive Interventional Therapy, Sun Yat-sen University Cancer Center, State Key Laboratory of Oncology in South China, Collaborative Innovation Center for Cancer Medicine, Guangzhou 510060, Guangdong, P. R. China. ${ }^{2}$ Department of Interventional Radiology, St. John's Medical Center, Tulsa, OK 74131, USA. ${ }^{3}$ Department of Oncologic Interventional Therapy, The First Affiliated Hospital, Sun Yat-sen University, Guangzhou 510080, Guangdong, P. R. China. ${ }^{4}$ Department of Radiology Intervention, Sun Yat-sen Memorial Hospital, Sun Yat-sen University, Guangzhou 510120, Guangdong, P. R. China. ${ }^{5}$ Department of Intervention, The Third Affiliated Hospital, Sun Yat-sen University, Guangzhou 510630, Guangdong, P. R. China.

\section{Acknowledgements}

This work was supported by grants from the National Natural Science Foundation of China (No. 81371652) and the Science and Technology Planning Project of Guangdong Province (No. 2012B031800120).

\section{Competing interests}

The authors declare that they have no any actual or potential competing interests.

Received: 20 October 2015 Accepted: 10 October 2016 Published online: 07 January 2017

\section{References}

1. Chen $W$, Zheng R, Zeng H, Zhang S. The incidence and mortality of major cancers in China, 2012. Chin J Cancer. 2016;35(1):73.

2. Cheung TT, Poon RT, Chok KS, Chan AC, Tsang SH, Dai WC, et al. Management of spontaneously ruptured hepatocellular carcinomas in the radiofrequency ablation era. PLoS ONE. 2014;9(4):e94453.

3. Lai EC, Lau WY. Spontaneous rupture of hepatocellular carcinoma: a systematic review. Arch Surg. 2006;141(2):191-8.

4. Liu CL, Ngan H, Lo CM, Fan ST. Ruptured hepatocellular carcinoma as a complication of transarterial oily chemoembolization. Br J Surg. 1998;85(4):512-4.

5. Kim JY, Lee JS, Oh DH, Yim YH, Lee HK. Transcatheter arterial chemoembolization confers survival benefit in patients with a spontaneously ruptured hepatocellular carcinoma. Eur I Gastroenterol Hepatol. 2012;24(6):640-5.

6. Ng KK, Lam CM, Poon RT, Law WL, Seto CL, Fan ST. Radiofrequency ablation as a salvage procedure for ruptured hepatocellular carcinoma. Hepatogastroenterology. 2003;50(53):1641-3.

7. Luo RG, Fao F, Huang JH, GU YK, Jiang XY, Huang YJ. Diluted hydrochloric acid generates larger radiofrequency ablation lesions in excised porcine livers. Diagn Interv Radiol (Ankara, Turkey). 2013;19(2):145-9.

8. Kanematsu M, Imaeda T, Yamawaki Y, Seki M, Goto H, Sone Y, et al. Rupture of hepatocellular carcinoma: predictive value of CT findings. Am J Roentgenol. 1992;158(6):1247-50.

9. Ong GB, Taw JL. Spontaneous rupture of hepatocellular carcinoma. Br Med J. 1972:4(5833):146-9.

10. Fuchizaki U, Miyamori H, Kitagawa S, Kaneko S. Radiofrequency ablation for life-threatening ruptured hepatocellular carcinoma. J Hepatol. 2004;40(2):354-5.

11. Leung KL, Lau WY, Lai PB, Yiu RY, Meng WC, Leow CK. Spontaneous rupture of hepatocellular carcinoma: conservative management and selective intervention. Arch Surg. 1999;134(10):1103-7.

12. Kim HC, Chung JW, Lee W, Jae HJ, Park JH. Recognizing extrahepatic collateral vessels that supply hepatocellular carcinoma to avoid complications of transcatheter arterial chemoembolization. Radiographics. 2005;25(Suppl 1):S25-39. 
13. Huang JH, Gao F, Gu YK, Li WQ, Lu LW. Combined treatment of hepatocellular carcinoma with partial splenic embolization and transcatheter hepatic arterial chemoembolization. World J Gastroenterol. 2007;13(48):6593-7.

14. Jiang XY, Gu YK, Huang JH, Gao F, Zou RH, Zhang TQ. Ex vivo liver experiment of hydrochloric acid-infused and saline-infused monopolar radiofrequency ablation: better outcomes in temperature, energy, and coagulation. Cardiovasc Intervent Radiol. 2016;39(4):600-5.

15. Weijian F, Zan L, Suhong H, Hongmei Z, Lei Z, Yanjie Z, et al. Destructive effect of percutaneous hydrochloric acid injection therapy for liver cancer-a preliminary experimental and clinical study. Gan To Kagaku Ryoho. 2006;33(12):1852-6.

\section{Submit your next manuscript to BioMed Central and we will help you at every step:}

- We accept pre-submission inquiries

- Our selector tool helps you to find the most relevant journal

- We provide round the clock customer support

- Convenient online submission

- Thorough peer review

- Inclusion in PubMed and all major indexing services

- Maximum visibility for your research

Submit your manuscript at

www.biomedcentral.com/submit 